\title{
Adverse Ocular Events following COVID-19 Vaccination
}

\author{
Taher K. Eleiwa ${ }^{1}$ - Eric D. Gaier ${ }^{2,3,4,5}$ - Abid Haseeb ${ }^{6} \cdot$ Reem H. ElSheikh $^{7}$ - Ahmed B. Sallam ${ }^{8}$. \\ Abdelrahman M. Elhusseiny ${ }^{8}$ (D)
}

Received: 28 August 2021 / Revised: 28 August 2021 / Accepted: 13 September 2021 / Published online: 24 September 2021 (c) The Author(s), under exclusive licence to Springer Nature Switzerland AG 2021

Keywords Corona virus · COVID $\cdot$ Vaccine $\cdot$ Optic neuropathy $\cdot$ Uveitis

To the Editor,

We read with interest the article "Acute reduction of visual acuity and visual field after Pfizer-BioNTech COVID-19 vaccine 2 nd dose" by Santovito and Pinna [1]. The timeline of events beginning after the vaccination supports that the exposure caused the patient's symptoms. However, little insight can be gained without a corroborative neuroophthalmic examination within the window that the patient exhibited symptoms.

The neurologic and ophthalmic manifestations of SARSCoV-2 (COVID-19) are well established. In one study, COVID-19 infection resulted in ocular manifestations in $1.4 \%$ of patients; a review of the literature indicates that these manifestations most frequently result in reactive

Responsible Editor: John Di Battista.

Abdelrahman M. Elhusseiny

ElhusseinyAbdelrahma@uams.edu

1 Department of Ophthalmology, Benha University, Benha, Egypt

2 Department of Ophthalmology, Boston Children's Hospital, Boston, MA, USA

3 Department of Ophthalmology, Massachusetts Eye \& Ear, Boston, MA, USA

4 Department of Ophthalmology, Harvard Medical School, Boston, MA, USA

5 Picower Institute for Learning and Memory, Massachusetts Institute of Technology, Cambridge, MA, USA

6 Department of Ophthalmology and Visual Sciences, University of Illinois at Chicago, Chicago, IL, USA

7 Department of Ophthalmology, Cairo University Hospitals, Cairo, Egypt

8 Department of Ophthalmology, Harvey and Bernice Jones Eye Institute, University of Arkansas for Medical Sciences, Little Rock, AR, USA inflammation of the eyelids, follicular conjunctivitis, eye redness, foreign body sensation, and eye discharge [2-7]. Regarding neuro-ophthalmologic manifestations, optic neuritis has been reported in several reports at various stages of COVID-19 infection [8-13]. Several case reports have reported on cranial nerve palsies, particularly oculomotor (third cranial) nerve and abducens (sixth cranial) nerve palsies presenting with diplopia and/or ophthalmoplegia [14-20]. However, these ophthalmologic and neuro-ophthalmologic manifestations are non-specific, so definitive attribution to a particular exposure is generally difficult. In the current case, the onset, course, and duration of the reported symptoms narrow the field of etiological possibilities to the inflammatory and vascular categories.

Optic neuritis may manifest with reduction of visual acuity and darkening of the visual field, accompanied by pain exacerbated with ocular movement. In a review of adverse ocular events from 2010 to 2020, optic neuritis was found to be the most common event associated with nine different vaccines with a mean onset of 10.8 days (range: 1 day-1 month) post-injection [21]. The mechanism underlying optic neuritis in the setting of vaccination is not well understood; previous studies have suggested molecular mimicry between myelin basic protein and viral proteins, epitope spreading, bystander activation, and superantigen activation as potential mechanisms [22-26]. Visual prognosis is generally favorable, but ideal management remains elusive [21, 27].

Regarding the case presented by Santovito and Pinna, the likelihood the patient had an optic neuropathy is low given the brief duration (hours) of symptoms. Transient visual obscurations associated with optic disc edema (even that secondary to increased intracranial pressure, i.e., papilledema) are another consideration, but these events tend to manifest on the order of seconds and are elicited by transient increases in intrathoracic/central venous pressure. 


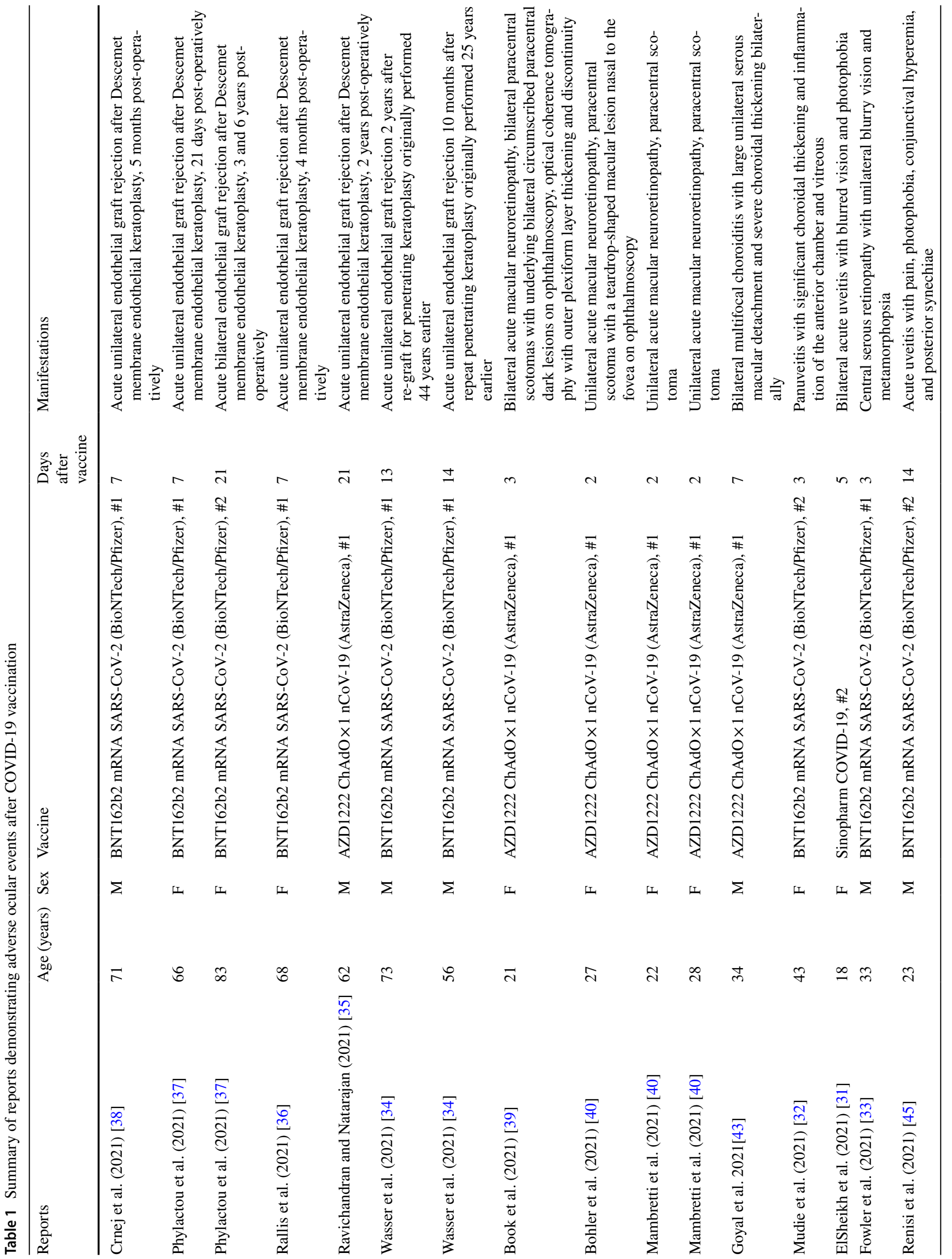


We cannot know if the patient had optic neuritis or optic disc edema that was present for longer than his symptoms without an examination.

Presumed inflammatory cranial neuropathies in general are common among patients after vaccines. We recently encountered a 46-year-old male patient who developed torsional, binocular diplopia 3 days after the second dose of Oxford-AstraZeneca ChAdOx1 nCoV-19 (AZD1222). Examination revealed a pattern of strabismus fitting that of a right 4 th cranial nerve palsy. Paretic ophthalmoplegia has also been reported with active COVID-19 infection. Falcone et al. reported a case of abducens cranial nerve palsy underlying binocular, horizonal diplopia in an otherwise healthy 32-year-old man 3 days after developing upper respiratory symptoms later confirmed to be COVID-19 infection [16]. Magnetic resonance imaging confirmed lateral rectus atrophy in the patient. Faucher et al. reported a case of oculomotor nerve palsy in an otherwise healthy 21-year-old man 2 weeks after COVID-19 infection manifesting after hospital discharge [28].

Intraocular inflammation is another possible mechanism by which the reported patient may have experienced his visual symptoms. Since February 2021, 46 reports of ocular side effects linked to the COVID-19 vaccine have been reported to the Vaccine Adverse Event Reporting System (VAERS) [21, 29]. The majority (74\%) involve the eyelid or conjunctiva [29, 30]. Inflammation of the optic nerve, retina, uveal tract, and anterior segment, combined to account for $9 \%$ of VAERS [30]. We recently reported a case of acute juvenile idiopathic arthritis-associated uveitis 5 days after the 2 nd dose of Sinopharm COVID-19 vaccine in an 18-year-old girl [31]. Panuveitis[32] and central serous retinopathy [33] have also been reported.

Despite the above-mentioned associations, the rapid resolution of visual symptoms over the course of hours argues against a uveitic process. It would be highly unusual for ocular inflammation to manifest and then self-resolve within hours. Photophobia, a hallmark symptomatic feature of uveitis, was not reported by the patient.

Table 1 summarizes our review of literature from 16 publications reporting on 20 patients with ocular complications following COVID-19 vaccinations. In brief, these included graft rejections [34-3831], macular neuroretinopathy [39-41], multifocal choroiditis [42, 43], acute uveitis [, $32,44,45]$, central serous retinopathy [33], arteritic anterior ischemic optic neuropathy [46], and acute zonal occult outer retinopathy [46].

In our opinion, the most likely explanation is that the patient suffered visual symptoms related to the central, systemic illness triggered by the vaccine. The presence of systemic symptoms (headache, dizziness and fatigue, and nausea) that accompanied and resolved with his visual symptoms reflect and support this hypothesis. Hypoperfusion 
of the retina might account for the peripheral visual loss, which self-corrected rapidly. Reduction of central acuity is less straight forward to explain, but can result from transient hypoperfusion of the retina, optic nerves, or any part of the visual pathways extending to the visual cortices. If this were the case, then we might not expect that any findings would have been apparent on even a complete dilated ophthalmic examination.

Ultimately, reporting of this patient's vaccine triggered very transient visual symptoms as described is of little value without a corroborating examination or objective evidence of ophthalmic pathology. The relevance of optic neuritis, inflammatory cranial neuropathies, and ocular inflammation and uveitis to this case is suspect for the same reason. No doubt there is a miriad of ways in which vaccines, particularly second doses or first doses in previous infected individuals, can affect the eyes and visual pathways to produce visual symptoms. Reporting of these symptoms as in the case of Santovito and Pinna is very commanded. However, reports of these adverse events should always be verified and supported by objective data from clinical examination and ancillary testing if a causal relationship is to be asserted.

\section{References}

1. Santovito LS, Pinna G. Acute reduction of visual acuity and visual field after Pfizer-BioNTech COVID-19 vaccine 2nd dose: a case report. Inflamm Res. 2021;70(9):931-3. https://doi.org/10.1007/ s00011-021-01476-9 (published Online First: Epub Date).

2. Wang D, Hu B, Hu C, et al. Clinical characteristics of 138 hospitalized patients with 2019 novel coronavirus-infected pneumonia in Wuhan, China. JAMA. 2020;323(11):1061-9. https://doi.org/ 10.1001/jama.2020.1585 (published Online First: Epub Date).

3. Ho D, Low R, Tong L, Gupta V, Veeraraghavan A, Agrawal R. COVID-19 and the ocular surface: a review of transmission and manifestations. Ocul Immunol Inflamm. 2020;28(5):726-34. https://doi.org/10.1080/09273948.2020.1772313 (published Online First: Epub Date).

4. Douglas KAA, Douglas VP, Moschos MM. Ocular manifestations of COVID-19 (SARS-CoV-2): a critical review of current literature. In Vivo. 2020;34(3 Suppl):1619-28. https://doi.org/10. 21873/invivo.11952 (published Online First: Epub Date).

5. Dockery DM, Rowe SG, Murphy MA, Krzystolik MG. The ocular manifestations and transmission of COVID-19: recommendations for prevention. J Emerg Med. 2020;59(1):137-40. https://doi.org/ 10.1016/j.jemermed.2020.04.060 (published Online First: Epub Date).

6. Eleiwa T, Abdelrahman SN, ElSheikh RH, Elhusseiny AM. Orbital inflammatory disease associated with COVID-19 infection. J AAPOS. 2021. https://doi.org/10.1016/j.jaapos.2021.04. 002 (published Online First: Epub Date).

7. Eleiwa TK, Elmaghrabi A, Helal HG, Abdelrahman SN, ElSheikh RH, Elhusseiny AM. Phlyctenular keratoconjunctivitis in a patient with COVID-19 infection. Cornea. 2021. https://doi.org/10.1097/ ico.0000000000002789 (published Online First: Epub Date).
8. Azab MA, Hasaneen SF, Hanifa H, Azzam AY. Optic neuritis post-COVID-19 infection. A case report with meta-analysis. Interdiscip Neurosurg. 2021;26:101320. https://doi.org/10.1016/j.inat. 2021.101320 (published Online First: Epub Date).

9. Benito-Pascual B, Gegundez JA, Diaz-Valle D, et al. Panuveitis and optic neuritis as a possible initial presentation of the novel coronavirus disease 2019 (COVID-19). Ocul Immunol Inflamm. 2020;28(6):922-5. https://doi.org/10.1080/09273948.2020.17925 12 (published Online First: Epub Date).

10. Leber HM, Sant'Ana L, Konichi da Silva NR, et al. Acute thyroiditis and bilateral optic neuritis following SARS-CoV-2 vaccination with CoronaVac: a case report. Ocul Immunol Inflamm. 2021. https://doi.org/10.1080/09273948.2021.1961815 (published Online First: Epub Date).

11. Parvez Y, Al Zarooni F, Khan F. Optic neuritis in a child with COVID-19: a rare association. Cureus. 2021;13(3):e14094. https://doi.org/10.7759/cureus.14094 (published Online First: Epub Date)

12. Sawalha K, Adeodokun S, Kamoga GR. COVID-19-induced acute bilateral optic neuritis. J Investig Med High Impact Case Rep. 2020;8:2324709620976018. https://doi.org/10.1177/2324709620 976018.

13. Sinha A, Dwivedi D, Dwivedi A, Bajaj N. Optic neuritis as a presenting symptom of post-COVID-19 multisystem inflammatory syndrome in children (MIS-C). Indian J Pediatr. 2021. https:// doi.org/10.1007/s12098-021-03921-3 (published Online First: Epub Date).

14. Lonardi V, Meneghesso D, Debertolis G, Pin JN, Nosadini M, Sartori S. Isolated third cranial nerve palsy and COVID-19 infection in a child. Pediatr Neurol. 2021;120:11. https://doi.org/10.1016/j. pediatrneurol.2021.03.011 (published Online First: Epub Date).

15. Francis JE. Abducens palsy and anosmia associated with COVID19: a case report. Br Ir Orthopt J. 2021;17(1):8-12. https://doi. org/10.22599/bioj.167 (published Online First: Epub Date).

16. Falcone MM, Rong AJ, Salazar H, Redick DW, Falcone $\mathrm{S}$, Cavuoto KM. Acute abducens nerve palsy in a patient with the novel coronavirus disease (COVID-19). J AAPOS. 2020;24(4):216-7. https://doi.org/10.1016/j.jaapos.2020.06.001 (published Online First: Epub Date).

17. Elenga N, Martin E, Gerard M, Osei L, Rasouly N. Unilateral diplopia and ptosis in a child with COVID-19 revealing third cranial nerve palsy. J Infect Public Health. 2021;14(9):1198-200. https://doi.org/10.1016/j.jiph.2021.08.007 (published Online First: Epub Date).

18. Douedi S, Naser H, Mazahir U, Hamad AI, Sedarous M. Third cranial nerve palsy due to COVID-19 infection. Cureus. 2021;13(4):e14280. https://doi.org/10.7759/cureus.14280 (published Online First: Epub Date).

19. Dinkin M, Gao V, Kahan J, et al. COVID-19 presenting with ophthalmoparesis from cranial nerve palsy. Neurology. 2020;95(5):221-3. https://doi.org/10.1212/WNL.0000000000 009700 (published Online First: Epub Date).

20. de Oliveira MR, Lucena A, Higino TMM, Ventura CV. Oculomotor nerve palsy in an asymptomatic child with COVID-19. J AAPOS. 2021;25(3):169-70. https://doi.org/10.1016/j.jaapos. 2021.02.001 (published Online First: Epub Date).

21. Cheng JY, Margo CE. Ocular adverse events following vaccination: overview and update. Surv Ophthalmol. 2021. https://doi. org/10.1016/j.survophthal.2021.04.001 (published Online First: Epub Date)

22. Nahill SR, Welsh RM. High frequency of cross-reactive cytotoxic $\mathrm{T}$ lymphocytes elicited during the virus-induced polyclonal cytotoxic T lymphocyte response. J Exp Med. 1993;177(2):317-27. https://doi.org/10.1084/jem.177.2.317 (published Online First: Epub Date). 
23. Barnaba V. Viruses, hidden self-epitopes and autoimmunity. Immunol Rev. 1996;152:47-66. https://doi.org/10.1111/j.1600065x.1996.tb00910.x (published Online First: Epub Date).

24. McCoy L, Tsunoda I, Fujinami RS. Multiple sclerosis and virus induced immune responses: autoimmunity can be primed by molecular mimicry and augmented by bystander activation. Autoimmunity. 2006;39(1):9-19. https://doi.org/10.1080/0891693050 0484799 (published Online First: Epub Date).

25. Miller SD, Vanderlugt CL, Begolka WS, et al. Persistent infection with Theiler's virus leads to CNS autoimmunity via epitope spreading. Nat Med. 1997;3(10):1133-6. https://doi.org/10.1038/ nm1097-1133 (published Online First: Epub Date).

26. Hull TP, Bates JH. Optic neuritis after influenza vaccination. Am J Ophthalmol. 1997;124(5):703-4. https://doi.org/10.1016/s00029394(14)70918-3 (published Online First: Epub Date).

27. Stubgen JP. A literature review on optic neuritis following vaccination against virus infections. Autoimmun Rev. 2013;12(10):990-7. https://doi.org/10.1016/j.autrev.2013.03.012 (published Online First: Epub Date).

28. Faucher A, Rey PA, Aguadisch E, Degos B. Isolated post SARSCoV-2 diplopia. J Neurol. 2020;267(11):3128-9. https://doi.org/ 10.1007/s00415-020-09987-x (published Online First: Epub Date).

29. US Centers for Disease Control and Prevention. CDC WONDER online database. Accessed August 10, 2021. https://wonder.cdc. gov/vaers.html

30. Cheng JY, Margo CE. Ocular adverse events following vaccination: overview and update. Survey oOphthalmol. 2021. https:// doi.org/10.1016/j.survophthal.2021.04.001 ([published Online First: Epub Date]).

31. ElSheikh RH, Haseeb A, Eleiwa TK, Elhusseiny AM. Acute uveitis following COVID-19 vaccination. Ocul Immunol Inflamm. 2021;1:3. https://doi.org/10.1080/09273948.2021.1962917 (published Online First: Epub Date).

32. Mudie LI, Zick JD, Dacey MS, Palestine AG. Panuveitis following vaccination for COVID-19. Ocul Immunol Inflamm. 2021. https:// doi.org/10.1080/09273948.2021.1949478 (published Online First: Epub Date).

33. Fowler N, Mendez Martinez NR, Pallares BV, Maldonado RS. Acute-onset central serous retinopathy after immunization with COVID-19 mRNA vaccine. Am J Ophthalmol Case Rep. 2021;23:101136. https://doi.org/10.1016/j.ajoc.2021.101136 (published Online First: Epub Date).

34. Wasser LM, Roditi E, Zadok D, Berkowitz L, Weill Y. Keratoplasty rejection after the BNT162b2 messenger RNA vaccine. Cornea. 2021;40(8):1070-2. https://doi.org/10.1097/ico.00000 00000002761 (published Online First: Epub Date).

35. Ravichandran $S$, Natarajan R. Corneal graft rejection after COVID-19 vaccination. Indian J Ophthalmol. 2021;69(7):19534. https://doi.org/10.4103/ijo.IJO_1028_21 ([published Online First: Epub Date]).

36. Rallis KI, Ting DSJ, Said DG, Dua HS. Corneal graft rejection following COVID-19 vaccine. Eye (Lond). 2021. https://doi.org/ 10.1038/s41433-021-01671-2 (published Online First: Epub Date).

37. Phylactou M, Li JO, Larkin DFP. Characteristics of endothelial corneal transplant rejection following immunisation with SARS-CoV-2 messenger RNA vaccine. Br J Ophthalmol. 2021;105(7):893-6. https://doi.org/10.1136/bjophthalmol-2021319338 (published Online First: Epub Date).

38. Crnej A, Khoueir Z, Cherfan G, Saad A. Acute corneal endothelial graft rejection following COVID-19 vaccination. J Fr Ophtalmol. 2021. https://doi.org/10.1016/j.jfo.2021.06.001 (published Online First: Epub Date).

39. Book BAJ, Schmidt B, Foerster AMH. Bilateral acute macular neuroretinopathy after vaccination against SARS-CoV-2. JAMA Ophthalmol. 2021;139(7):e212471. https://doi.org/10.1001/jamao phthalmol.2021.2471 (published Online First: Epub Date).

40. Mambretti M, Huemer J, Torregrossa G, Ullrich M, Findl O, Casalino G. Acute macular neuroretinopathy following coronavirus disease 2019 vaccination. Ocul Immunol Inflamm. 2021. https://doi.org/10.1080/09273948.2021.1946567 (published Online First: Epub Date).

41. Bohler AD, Strom ME, Sandvig KU, Moe MC, Jorstad OK. Acute macular neuroretinopathy following COVID-19 vaccination. Eye (Lond). 2021. https://doi.org/10.1038/s41433-021-01610-1 (published Online First: Epub Date).

42. Mungmunpuntipantip R, Wiwanitkit V. COVID-19 vaccination and bilateral multifocal choroiditis. Ocul Immunol Inflamm. 2021. https://doi.org/10.1080/09273948.2021.1968003 (published Online First: Epub Date).

43. Goyal M, Murthy SI, Annum S. Bilateral multifocal choroiditis following COVID-19 vaccination. Ocul Immunol Inflamm. 2021. https://doi.org/10.1080/09273948.2021.1957123 (published Online First: Epub Date).

44. Rabinovitch T, Ben-Arie-Weintrob Y, Hareuveni-Blum T, et al. Uveitis following the BNT162b2 mRNA vaccination against SARS-CoV-2 infection: a possible association. Retina. 2021. https://doi.org/10.1097/iae.0000000000003277 (published Online First: Epub Date).

45. Renisi G, Lombardi A, Stanzione M, Invernizzi A, Bandera A, Gori A. Anterior uveitis onset after bnt $162 \mathrm{~b} 2$ vaccination: is this just a coincidence? Int J Infect Dis. 2021;110:95-7. https://doi. org/10.1016/j.ijid.2021.07.035 (published Online First: Epub Date).

46. Maleki A, Look-Why S, Manhapra A, Foster CS. COVID19 recombinant mrna vaccines and serious ocular inflammatory side effects: real or coincidence? J Ophthalmic Vis Res. 2021;16(3):490-501. https://doi.org/10.18502/jovr.v16i3.9443 (published Online First: Epub Date).

Publisher's Note Springer Nature remains neutral with regard to jurisdictional claims in published maps and institutional affiliations. 